\title{
Analisi della funzione tiroidea in 50 pazienti con COVID-19: uno studio retrospettivo
}

\author{
Nicola Viola ${ }^{1} \cdot$ Francesco Latrofa $^{1}$
}

Accettato: 27 dicembre 2020 / Pubblicato online: 23 agosto 2021

(c) The Author(s) 2021

\section{Commento a:}

Thyroid function analysis in 50 patients with

COVID-19: a retrospective study.

M. Chen, W. Zhou, W. Xu.

Thyroid (2021) 31(1):8-11

L'infezione da SARS-CoV-2, responsabile della COVID19 , ha interessato oltre 200 paesi nei 6 diversi continenti. Sebbene sia ormai noto che si tratti di una malattia con coinvolgimento multi-organo, al momento non è stato chiaramente definito l'impatto della COVID-19 sulla funzione tiroidea.

Lo scopo di questo studio è stato quello di valutare retrospettivamente la funzione tiroidea in pazienti con COVID19. Tale studio ha riguardato 50 individui cinesi affetti da COVID-19 e privi di storia di malattie della tiroide nei quali sono stati valutati i livelli sierici di tiroxina totale (TT4), triiodotironina totale (TT3) e ormone stimolante la tiroide (TSH) nel corso di COVID-19 e dopo la guarigione. I gruppi di controllo erano costituiti da soggetti sani e pazienti con polmonite non sostenuta da SARS-CoV-2. Nei pazienti affetti da COVID-19 sono stati riscontrati livelli inferiori sia di TT3 che di TSH rispetto ai controlli, mentre non è stata rilevata alcuna differenza riguardo i valori di TT4. Inoltre, gli autori hanno osservato una correlazione inversa tra la gravità del COVID-19 e i livelli di TT3 e TSH. Dopo la guarigione non è stata osservata alcuna differenza nei test di funzionalità tiroidea tra i pazienti con COVID-19 e quelli dei gruppi di controllo. Questi risultati sono indicativi della presenza di una non-thyroidal illness syndrome (NTIS) tipica di molti

F. Latrofa

francesco.latrofa@unipi.it

1 Dipartimento di Medicina Clinica e Sperimentale, Università di Pisa, Pisa, Italia stati patologici con coinvolgimento sistemico e che si risolve con la guarigione.

Tuttavia, il fatto che i livelli sierici di TSH dei pazienti con COVID-19 fossero significativamente più bassi rispetto ai pazienti con polmonite non COVID-19 con un simile grado di gravità potrebbe indicare un'inibizione nei confronti delle cellule ipofisarie TSH-secernenti mediante un meccanismo virale diretto o mediato dall'attivazione di varie citochine proinfiammatorie o legato alle alterazioni dei feedback ormonali indotti dai trattamenti farmacologici, come i glucorticoidi assunti da 31/50 dei pazienti con COVID-19 valutati in questo studio.

Funding Note Open access funding provided by Università di Pisa within the CRUI-CARE Agreement.

Nota della casa editrice Springer Nature rimane neutrale in riguardo alle rivendicazioni giurisdizionali nelle mappe pubblicate e nelle affiliazioni istituzionali.

Open Access This article is licensed under a Creative Commons Attribution 4.0 International License, which permits use, sharing, adaptation, distribution and reproduction in any medium or format, as long as you give appropriate credit to the original author(s) and the source, provide a link to the Creative Commons licence, and indicate if changes were made. The images or other third party material in this article are included in the article's Creative Commons licence, unless indicated otherwise in a credit line to the material. If material is not included in the article's Creative Commons licence and your intended use is not permitted by statutory regulation or exceeds the permitted use, you will need to obtain permission directly from the copyright holder. To view a copy of this licence, visit http://creativecommons.org/licenses/by/4.0/. 